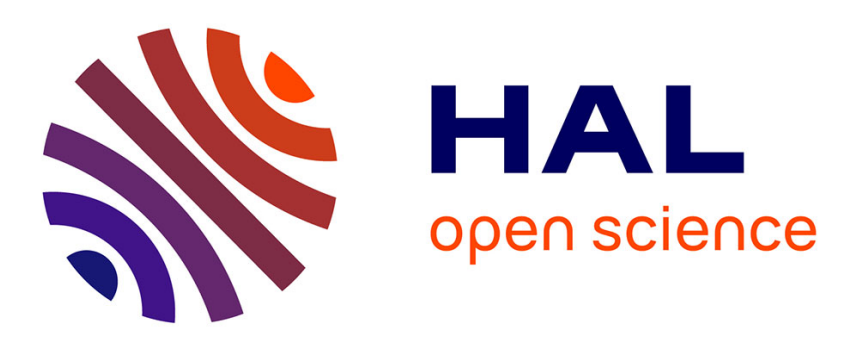

\title{
Reversal of degradation of information masks in lithium niobate
}

\author{
D. Sando, E. Jaatinen, F. Devaux
}

\section{To cite this version:}

D. Sando, E. Jaatinen, F. Devaux. Reversal of degradation of information masks in lithium niobate. Applied optics, 2009, 48 (24), pp.4676-4682. 10.1364/AO.48.004676 . hal-00411245

\section{HAL Id: hal-00411245 \\ https://hal.science/hal-00411245}

Submitted on 23 Apr 2021

HAL is a multi-disciplinary open access archive for the deposit and dissemination of scientific research documents, whether they are published or not. The documents may come from teaching and research institutions in France or abroad, or from public or private research centers.
L'archive ouverte pluridisciplinaire $\mathbf{H A L}$, est destinée au dépôt et à la diffusion de documents scientifiques de niveau recherche, publiés ou non, émanant des établissements d'enseignement et de recherche français ou étrangers, des laboratoires publics ou privés. 


\title{
Reversal of degradation of information masks in lithium niobate
}

\author{
Daniel Sando, ${ }^{1, \star}$ Esa Jaatinen, ${ }^{1}$ and Fabrice Devaux ${ }^{2}$ \\ ${ }^{1}$ Applied Optics and Nanotechnology Program, Queensland University of Technology, \\ GPO Box 2434, Brisbane, Queensland 4001, Australia \\ 2Département d'Optique P. M. Duffieux, Institut Franche-Comté Electronique, Mécanique, \\ Thermique et Optique - Sciences et Technologies, UMR CNRS 6174, \\ Université de Franche-Comté, 16 route de Gray, \\ F-25030 Besançon Cedex, France \\ ${ }^{*}$ Corresponding author: d.sando @ qut.edu.au
}

\begin{abstract}
We report on the reversal of degradation of information masks stored in self-defocusing lithium niobate. After a long writing time, the image degradation appears as the splitting of refractive-index patterns stored in the medium. The reversal is achieved simply by illuminating the crystal with incoherent light from a halogen lamp. The reversal occurs because the refractive-index changes responsible for the splitting are of a smaller magnitude and are therefore erased first during incoherent illumination. Additionally, we gain insight into the storage, degradation, and erasure dynamics using a timedependent numerical model of the photorefractive effect in this medium. Since the data can be recovered from a degraded state in which the original data are unrecognizable, this technique could be utilized in such applications as image scrambling or encryption.
\end{abstract}

\section{Introduction}

Lithium niobate is an important optical material that has been used for more than 40 years to modulate optical fields. Its novel nonlinear optical properties have led to various other practical applications such as frequency conversion [1], optical data storage [2], solitons [3], optical wave mixing, and optical circuitry $[4,5]$. The application of lithium niobate in the field of 3D data storage is particularly promising. Since the light-induced refractive-index changes are erasable, this material is well suited to rewritable data storage [6]. For more permanent storage, the data can be thermally fixed [7]; this is achieved by heating the crystal during or after recording. Alternatively, as used in this study, nondestructive readout can be achieved by reading at a wavelength to which the material is not sensitive.

An interesting property of this material is the phenomenon of self-defocusing and focusing [8]. This effect arises for a beam propagating through the medium whereby it self-diffracts from the lightinduced refractive-index changes. In lithium niobate the effect can be self-focusing (as used for bright solitons $[9,10]$ ) or self-defocusing (as used for dark solitons [3]).

Although the use of this material to store holographic data is well known, recently there has been much interest and progress made in using lithium niobate to store binary [11] or digital data [12]. In these applications, light-induced regions of low refractive index act as the data bits and significant storage capacity is possible if the volume occupied by each bit is small and the volume of the lithium niobate medium is large. The information is retrieved by illuminating the medium with a nonerasing readout 
beam that is imaged to reveal the regions of bright and dark that correspond to the individual binary data bits.

To store information in this way, care must be taken to avoid degrading the fidelity of the data in the writing process because of the self-defocusing effect of the lithium niobate medium. Here we show that, even at low cw writing intensities, this effect can completely scramble the readout image to a point at which the original data are unrecognizable. Specifically, we present the effects of image degradation on data stored as information masks in a self-defocusing lithium niobate crystal. After an exposure time that is longer than is necessary to write the patterns clearly, the stored information is degraded; the data bits (vertical stripes in this case) split into finer filaments. The degradation is caused by the writing field being diffracted from the already stored structures and inducing further, more complicated, refractive-index changes.

In what we believe to be the first report of this behavior, we show that the reversal of this splitting process is easily achieved by incoherent illumination of the crystal, allowing the initial data to be retrieved. Here the erasure restores the original pattern by removing the refractive-index changes that correspond to the complicated structures first, because they are of smaller magnitude. The dynamics of the splitting process observed during writing are seen to follow the reverse progression, back to a well-defined image that eventually fades under continued incoherent illumination. As will be discussed, the observed behavior agrees well with a detailed numerical model. The effect of the feature size on the data scrambling and retrieval process is also discussed. Furthermore, Fourier correlation analysis is used to show that indeed the degradation process is reversed. Since the data can be retrieved from a degraded state in which it is almost unrecognizable, this technique could find application in image scrambling or encryption.

\section{Experiment}

The experimental setup is shown in Fig. 1. An amplitude mask (shown in Fig. 2) consisting of transparent stripes on an opaque background, is illuminated by an expanded and collimated $532 \mathrm{~nm}$ frequencydoubled Nd:YAG beam $(10 \mathrm{~mW}$; extraordinary polarization) and focused onto the front face of the lithium niobate crystal $[3 \mathrm{~mm} \times 10 \mathrm{~mm} \times 8 \mathrm{~mm}(z \times x \times y)$ congruently melted, $0.05 \%$ Fe doped]. The intensity of the writing beam at the crystal is $40 \mathrm{~mW} / \mathrm{cm}^{2}$. There

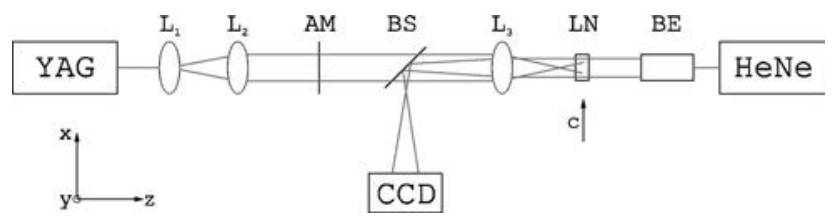

Fig. 1. Experimental setup: YAG, Nd:YAG laser at $532 \mathrm{~nm}$; AM, amplitude mask; BS, beam splitter; $\mathrm{LN}, \mathrm{LiNbO}_{3}$ crystal ( $c$ axis in the $x$ direction); $\mathrm{BE}$, beam expander; $\mathrm{HeNe}, \mathrm{He}-\mathrm{Ne}$ laser at $633 \mathrm{~nm}$; CCD, CCD camera attached to computer.

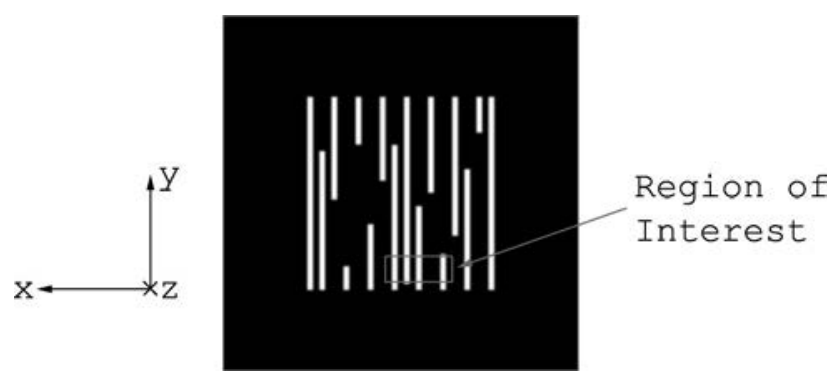

Fig. 2. Input amplitude mask of clear stripes (white) on an opaque background (black).

is no applied field to the crystal (open-circuit configuration), i.e., the crystal is in the defocusing regime. The field profile of the writing beam as it propagates through the crystal is relatively complex because of both diffraction and nonlinear effects, with the latter occurring after some exposure time. This method of information mask storage in a photorefractive crystal is similar in principle to those previously reported [13]; however, because we are dealing with a smaller spatial periodicity and a thicker sample, the beam structure through the crystal is considerably more complex.

The refractive index is photomodified when in regions with high intensity (bright regions) electrons are excited from their traps into the conduction band. The charges migrate as a result of the photovoltaic field, creating a nonuniform charge distribution. This nonuniform distribution of charges induces a spacecharge (SC) field and the refractive index is modified by the linear electro-optic effect [14]. The modulation of the writing field intensity is in the $x$ direction (i.e., the stripes are vertical not horizontal) because SC fields induced in the direction of the $c$ axis cause a larger $\Delta n$ than SC fields perpendicular to the $c$ axis. The induced refractive-index changes can persist in this medium for many days, depending on the dark conductivity and background illumination.

Readout of the data is achieved with a $2 \mathrm{~mW}$ $633 \mathrm{~nm} \mathrm{He}-\mathrm{Ne}$ beam, expanded so that its intensity is $0.6 \mathrm{~mW} / \mathrm{cm}^{2}$. The readout beam counterpropagates through the crystal relative to the writing beam; this allows easy monitoring of the readout while writing without beam switching. A readout beam at $633 \mathrm{~nm}$ is used because it does not significantly erase the stored pattern, since the material is practically insensitive at this wavelength at these low intensities [15]. The readout process itself is based on the guiding of the initially uniform readout beam by the refractive-index structures in the medium. As the beam propagates through the crystal, its intensity profile is modified by these refractive-index changes. The readout process is monitored with a CCD camera placed at the image plane of the front face of the crystal. This allows a representation of the patterns stored in the crystal to be monitored on the CCD camera.

Degradation of the stored pattern occurs after an extended writing time, when the refractive-index changes in the medium begin to have an appreciable 
effect on the propagation of the writing beam. Typical readout images taken by the CCD camera during the writing process for a bit width of $36 \mu \mathrm{m}$ are shown in Figs. 3(a)-3(c). After 2 min exposure to the writing beam, the bits are well defined with high contrast; after 5 min each bit has split into a number of smaller filaments; and after 40 min the pattern has become almost unrecognizable. After the writing process is complete, the write beam is turned off and two $50 \mathrm{~W}$ halogen lamps are used to erase the refractive-index pattern. During this time the red readout beam is monitored with the CCD camera.

The readout image at various times during the erasure process is shown in Figs. 3(d)-3(f). Here it is shown that the image degradation process has undergone a reversal with the evolution of the stored data retracing the steps that occurred during writing. The most interesting feature here is that, qualitatively speaking, the initial pattern is restored from the degraded one [Fig. 3(e)]. To analyze the data storage and erasure processes, on each image we take an intensity cross section through a defined region of interest (ROI) as indicated in Fig. 2. The data can then be read from the profile as a series of peaks and troughs. We define the average horizontal profile of the ROI indicated in Fig. 2 as

$$
I_{\text {av }}(m)=\frac{1}{N} \sum_{n} I(m, n) ; m=1,2, \ldots, M
$$

where $I(m, n)$ is the pixel value at row $n$ and column $m, N$ is the number of rows, and $M$ is the number of columns of the ROI. $I_{\mathrm{av}}$ for the ROI for the experimental data is plotted in Figs. 4(a)-4(c) for various times after writing begins. Peaks 1-4 indicated in
Fig. 4(a) split into a number of smaller filaments after $5 \mathrm{~min}$, as shown in Fig. 4(b). After further illumination, the degradation of the patterns is so extensive that the bits are no longer distinguishable [Fig. 4(c)].

The average profiles $I_{\mathrm{av}}$ in the ROI for the images shown in Figs. 3(c)-3(e) during the erasure process are shown in Figs. 4(c)-4(e). The profiles in Fig. 4 clearly show that the original patterns are accurately restored during erasure [see, for example, Fig. 4(e)], in the reverse order in which they appeared during writing. Figure 4(f) shows the erasure process when it is further along; by this stage the incoherent light has begun to fade the refractive-index variations and the image quality is diminished.

The dimensions of the features on the mask were chosen so as to be clearly evident in the readout image. The whole writing and erasure process was repeated for a number of different feature sizes (i.e., the width and spacing of each stripe in the pattern) by changing the value of $L_{3}$ and adjusting distances to ensure that the crystal was always in the image plane. Feature sizes of $18,36,73$, and $146 \mu \mathrm{m}$ were investigated.

\section{Numerical Modeling}

Due to the complexity of the nonlinear photorefractive effect, in most applications it is difficult to derive an analytical solution that explains the actual observations. Generally it is necessary to make use of numerical models to describe experimental results. This is particularly true for complex illumination patterns such as the one used in this work. The photorefractive effect in lithium niobate is well described in most cases by the one-center model [16], which incorporates a source of charges (known as donors), and the same centers, once ionized, are

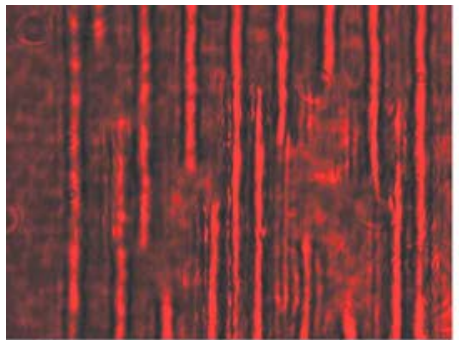

(a)

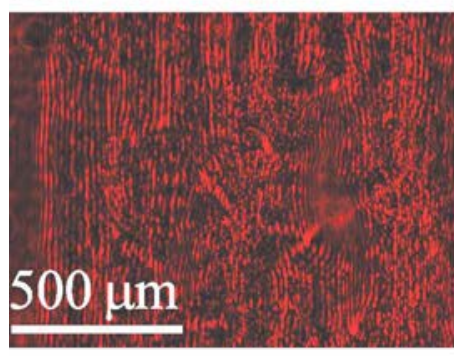

(d)

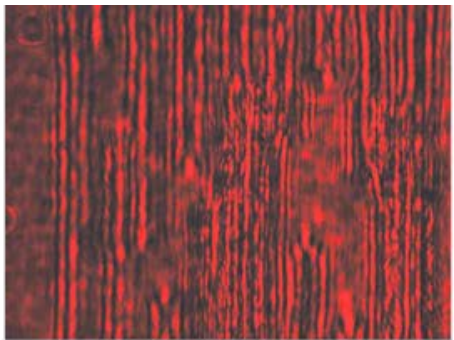

(b)

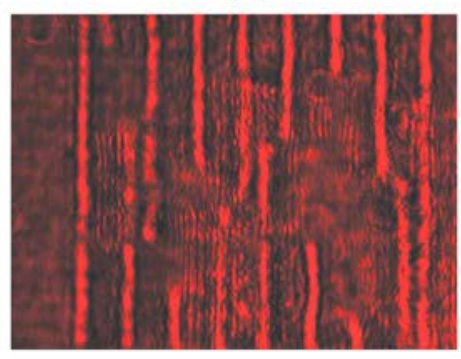

(e)

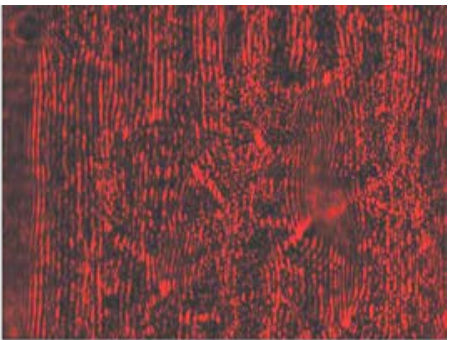

(c)

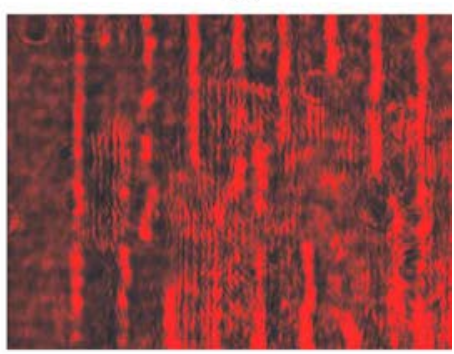

(f)

Fig. 3. (Color online) Images during the writing and erasure process: (a)-(c) during writing, (d)-(e) during erasure. The time of exposure to the writing beam is (a) $2 \mathrm{~min}$, (b) $5 \mathrm{~min}$, (c) $40 \mathrm{~min}$. The time from the start of erasure is (d) $1 \mathrm{~min}$, (e) $72 \mathrm{~min}$, (f) $97 \mathrm{~min}$. 


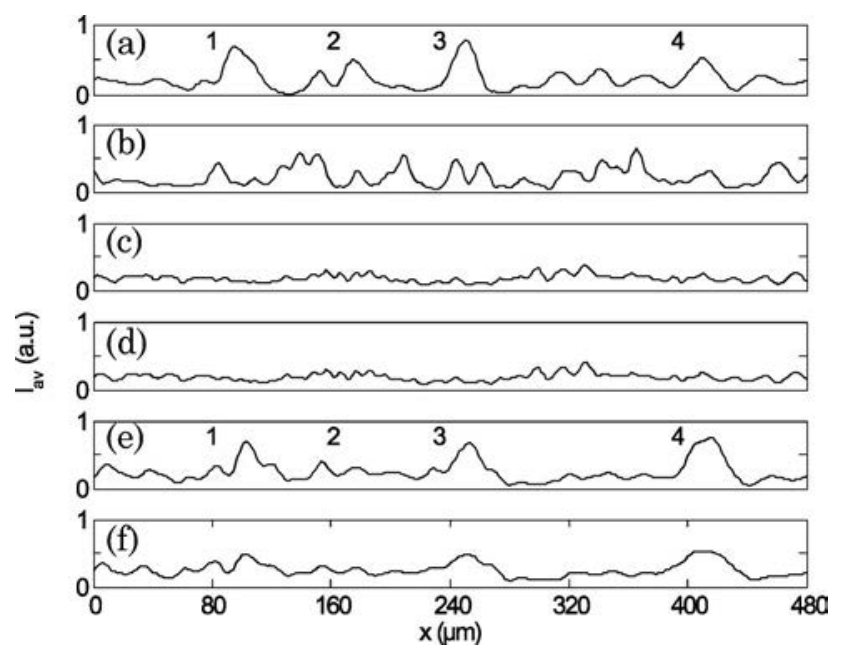

Fig. 4. Profiles $I_{\mathrm{av}}$ for the ROI of the images shown in Fig. 3. Exposure time to the writing beam is (a) $2 \mathrm{~min}$, (b) $5 \mathrm{~min}$, (c) $40 \mathrm{~min}$; time from the start of erasure is (d) $1 \mathrm{~min}$, (e) $72 \mathrm{~min}$, (f) $97 \mathrm{~min}$.

responsible for retrapping the charges from the conduction band. After illumination to a field of appropriate wavelength and intensity, the charges can be redistributed (driven by a photovoltaic field) and therefore create a nonuniform charge distribution. The induced SC field causes a change in the material's refractive index by the Pockels effect [14].

A detailed time-dependent three-dimensional model of the photorefractive effect in materials described by a one-center model was recently reported by Devaux et al. [17]. This model uses the standard one-center model equations (see, for example, [16]) to calculate the charge, donor, and acceptor densities in the medium. From this information the 3D SC field is calculated and thus the electric field is deduced in the medium. The change in refractive index is then calculated using the Pockels effect relationship that applies for lithium niobate under the experimental conditions used here:

$$
\Delta n=-\frac{1}{2} n_{e}^{3} r_{33} E_{x}
$$

where $n_{e}$ is the extraordinary refractive index, $r_{33}$ is the component of the electro-optic tensor, and $E_{x}$ is the $x$ component of the electric field. Only the $x$ component is used because the $y$ component of the field does not significantly alter the refractive index. The complete details of the model have been published elsewhere [17]. This model has successfully explained the observed temporal and steady-state photorefractive behavior in lithium niobate [17] as well as indium phosphide (InP:Fe) [18]. Here we use the model to gain insight into our experimental results. Note in our case that the intensity modulation is only in the $x$ direction and, therefore, a $2 \mathrm{D}$ treatment would suffice. Nonetheless, the 3D model used here will still give accurate results despite the superfluous transverse coordinate.

Within the specific context of our experiment, the modeling process is as follows. The writing beam, with its amplitude spatially modulated by the mask shown in Fig. 2, illuminates the front face of the lithium niobate medium. Simultaneously, the plane wave read beam counterpropagates through the medium, however, it does not play a role in redistributing charges or in modifying the index. At each time step the charge distribution, SC field, and index modification are calculated based on the effects of the write beam in the medium. Following this, the nonlinear propagation of the write and read beams is calculated in the modulated medium using the split-step Fourier method. Time is incremented and the process is repeated until the desired exposure time is attained.

Erasure of the index modulations in the medium is achieved in the model by removing the write field and increasing the dark intensity to simulate incoherent illumination. This effectively increases the thermal generation rate of the carriers uniformly in the medium, thus allowing them to redistribute. The red beam is still used to probe the index variations during the erasure process. At each time step, the new charge distribution and hence electric fields in the medium are calculated and the process continues until the desired erasure is obtained.

The readout beam after propagating through the crystal is shown in Fig. 5 at various instants of time during the writing and the erasure process. Here the data degradation has qualitatively the same appearance as in the experimental results, with each bit splitting into smaller filaments [Fig. 5(b)] and eventually breaking up completely [Fig. 5(c)]. The erasure [Figs. 5(d) and 5(e)] results in the recovery of the original data, with the filamentation progressing in approximately the reverse fashion when compared with the writing process. The agreement here between experiment and model is quite good.

The numerical model can also help us gain insight into the characteristics of the write and read beams as they propagate through the crystal. This is particularly useful as we have no simple way to determine this from the experiment. In Fig. 6 the field intensity of the write beam at the center of the pattern $(y=0)$ is plotted versus the propagation distance. Here it is evident that interactions between adjacent stripes in the write beam are likely to have a significant effect on the dynamics of the writing and erasure process, as will be discussed further.

\section{Discussion}

The feature size (i.e., stripe width) of the pattern has a significant effect on the dynamics of the data degradation process. Generally, for a constant writing beam intensity, the smaller the features, the shorter the exposure time required for the patterns to degrade, and the more severe the effects on the readout image. For example, for feature sizes of $\sim 146 \mu \mathrm{m}$, the splitting process in the pattern takes a longer time ( $\sim 19 \mathrm{~min}$ ) to appear, and the resultant steady-state image is somewhat similar to the original pattern, which is shown in Figs. 7(a) and 7(b). If, however, 


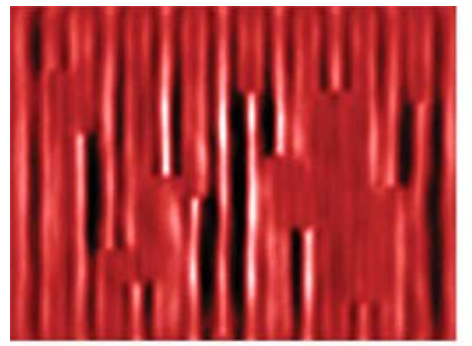

c

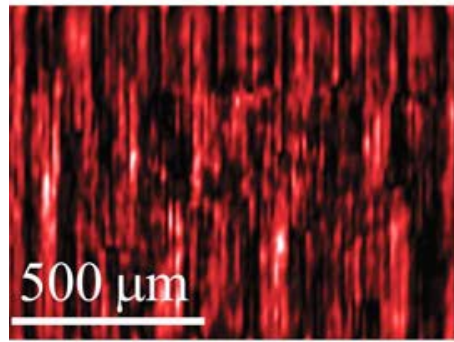

(d)

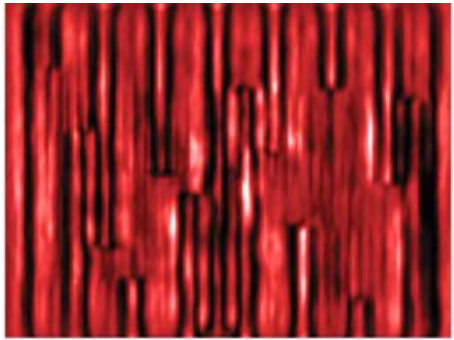

(b)

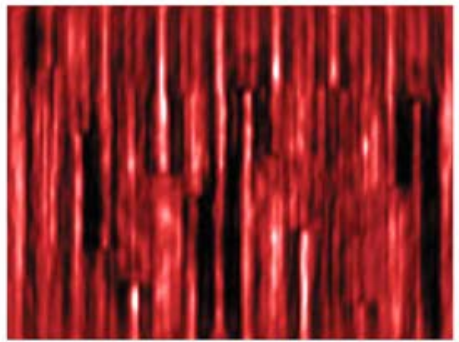

(e)

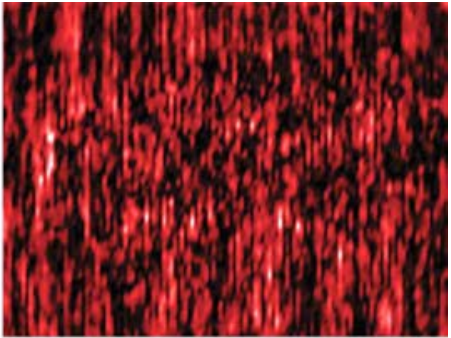

(c)

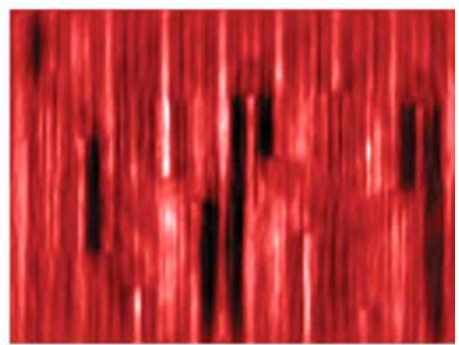

(f)

Fig. 5. (Color online) Readout images from the numerical model during writing and erasure: (a)-(c) during writing; (d)-(e) during erasure. The time of exposure to the writing beam corresponds to the times from Fig. 3, i.e., (a) $2 \mathrm{~min}$, (b) $5 \mathrm{~min}$, (c) $40 \mathrm{~min}$. The time from start of erasure is (d) $1 \mathrm{~min}$, (e) $72 \mathrm{~min}$, (f) $97 \mathrm{~min}$.

the feature size is reduced to $\sim 18 \mu \mathrm{m}$, the splitting takes place at a considerably earlier time (less than $2 \mathrm{~min}$ ) and the resultant steady-state pattern is extremely complex and unrecognizable in comparison with the original image [Figs. 7(c) and 7(d)]. Thus there is an optimal feature size for the trade-off between image scrambling and capability of recovering the scrambled image. This optimal size, based on our experiment, is approximately $35 \mu \mathrm{m}$.

A quantitative measure of the pattern degradation and recovery was obtained using a Fourier correlation (FC) of the readout image with the original mask shown in Fig. 2. We define the FC as

$$
\mathrm{FC}=\left|\sum_{k=0}^{M-1} \sum_{l=0}^{N-1} X(k, l) \circ Y(k, l)\right|,
$$

where $A \circ B$ is defined as the Hadamard product [19] (entrywise multiplication) of matrices $A$ and $B$, and $X(k, l)$ and $Y(k, l)$ are the $2 \mathrm{D}$ discrete Fourier transforms (DFTs) of the mask and readout images, respectively, with the DFT defined as

$$
F(k, l)=\sum_{n=0}^{N-1} \sum_{m=0}^{M-1} x(m, n) \exp \left(-j 2 \pi\left(\frac{k m}{M}+\frac{l n}{N}\right)\right),
$$

where $M$ and $N$ are the height and width, respectively, of the readout and mask images. This formulation, when applied to each readout image during write-erase, gives a single scalar for each time step, which is essentially a measure of how well the readout image matches the desired pattern. It would be expected that, when the stored pattern is at its maximum quality, the FC should show a peak. During the degradation process, we expect that the FC should decrease to a minimum value and during recovery it should increase as the pattern is restored.

The FC over the write-erase cycle for the $36 \mu \mathrm{m}$ feature size is shown in Fig. 8. Here we see that the $\mathrm{FC}$ reaches a maximum value at $t=2 \mathrm{~min}$; as the pattern degrades because of overexposure, the FC decreases rapidly. The small peak at $t=7 \mathrm{~min}$ corresponds to the situation in which each stripe has split into exactly two substripes. In this case there is a higher level of correlation in the stripes, resulting in a smaller peak. The same reasoning can be applied to the subpeak at $t=40 \mathrm{~min}$; however in this case each substripe has itself split into two additional stripes.

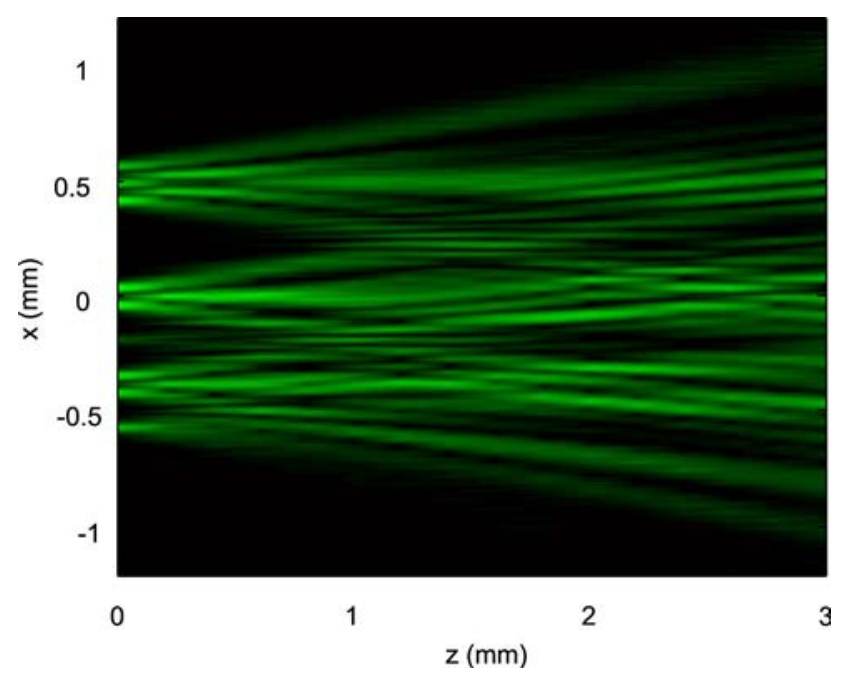

Fig. 6. (Color online) Propagation of the write beam at the start of the writing process showing a complicated beam structure. 


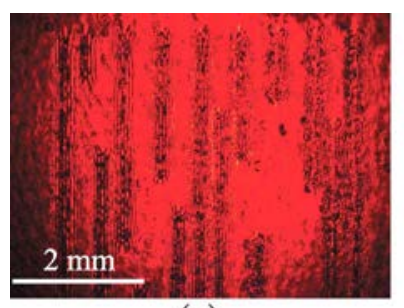

(a)

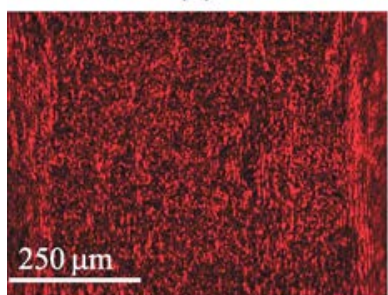

(c)

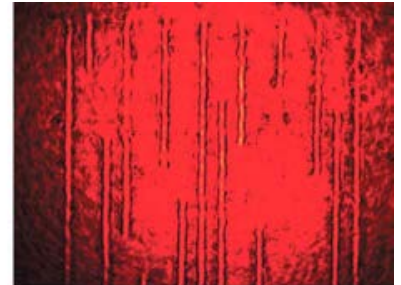

(b)

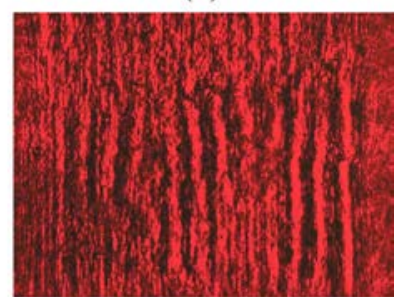

(d)
Fig. 7. (Color online) Readout images for different feature sizes: (a) degraded and (b) recovered images for the $146 \mu \mathrm{m}$ feature size; (c) degraded and (d) recovered images for the $18 \mu \mathrm{m}$ feature size.

The erasure process shows a reversal in the FC: a subpeak at $t=64 \mathrm{~min}$ is seen, followed by a peak at $t=115 \mathrm{~min}$, corresponding to the recovery of the degraded pattern. As expected, this peak is lower in magnitude than the original maximum FC attained during the writing process, which is due to the incoherent erasure influencing all regions of the crystal nonpreferentially, including the desired pattern information. As incoherent erasure continues, the FC diminishes as the refractive-index changes corresponding to the desired pattern are neutralized. Nonetheless, the FC is a valuable measure of pattern recovery and makes clear the time of incoherent erasure required for optimal recovery of the scrambled pattern. For the data presented in Fig. 8, the optimal erasure time is $72 \mathrm{~min}$. Any erasure longer than this will cause a reduction in the quality of the desired pattern. The oscillatory behavior of the FC during

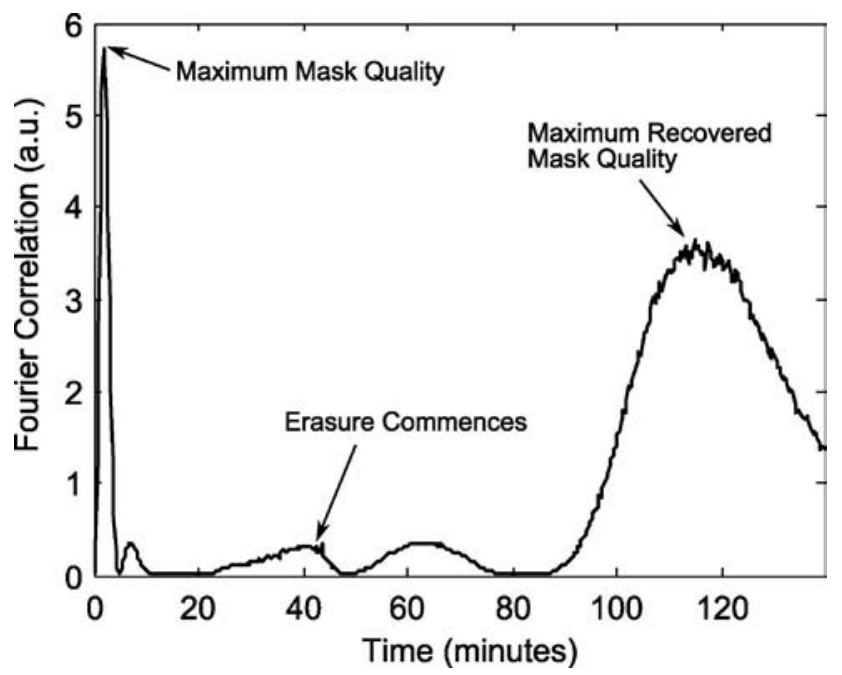

Fig. 8. FC of the readout image over the write and erase processes clearly showing that the information mask is scrambled and then recovered. write-erase is intriguing and is the subject of ongoing research.

Reversal of the degradation process occurs because the self-defocusing of the medium causes the writing field to spread further as the writing process evolves, lowering the intensity of the writing field. As a result, the refractive-index changes that are formed at later stages of the writing process are weaker than those produced when the writing beam is first introduced. Therefore, when erasure occurs with a low intensity incoherent source, the weaker changes are erased first leaving the stronger refractive changes corresponding to the original image.

The difficulty in recovering the original image for smaller feature sizes $(18 \mu \mathrm{m})$ is likely due to the writing beam profile being too complex or scrambled. As the feature size is decreased, the diffraction and nonlinear effects in the material become more pronounced, and this results in a write beam that loses all structure after a brief propagation distance inside the medium. This lack of structure is translated into the refractive-index distribution and therefore makes retrieval of initial patterns impossible. Indeed, this observation is consistent with the minimum distance between features of $25 \mu \mathrm{m}$ that is required for reasonable readout quality as determined by Gao et al. [20].

\section{Conclusions}

In conclusion, we have presented evidence of the reversal of image degradation of information masks in lithium niobate. Such degradation can be problematic for applications in which binary or digital data are stored in a photorefractive medium and occurs when the write time for the data is excessively long for the particular writing intensity used. However, shown here is a simple technique by which the degradation can be reversed allowing the original information to be retrieved. The reversal is induced simply by incoherent illumination of the crystal, and allows the recovery of a desired pattern from a state of considerable complexity. A detailed three-dimensional time-dependent numerical model for photorefractive materials was used to analyze the photorefractive behavior and was in good agreement with the observations. The model showed that a minimum feature size exists for any specific set of experimental conditions below which it is difficult to reverse the degradation. The modeling also showed that the reversal of the degradation is possible when the scrambled portions of the pattern produce refractive-index changes that are smaller in magnitude than those produced by the actual features themselves. Thus, during incoherent erasure, the smaller refractiveindex changes are erased first, leaving behind the stronger original patterns. Since the stored patterns can be scrambled to an almost unrecognizable state using this method and can still be recovered adequately, this technique could be applied to an application involving image scrambling or encryption. 


\section{References}

1. G. I. Stegeman and C. T. Seaton, "Nonlinear integrated optics," J. Appl. Phys. 58, R57-R78 (1985).

2. F. Mok, "Angle-multiplexed storage of 5000 holograms in lithium niobate," Opt. Lett. 18, 915-917 (1993).

3. M. Taya, M. C. Bashaw, M. M. Fejer, M. Segev, and G. C. Valley, "Observation of dark photovoltaic spatial solitons," Phys. Rev. A 52, 3095-3100 (1995).

4. O. Matoba, K. Itoh, and K. Kuroda, "Photorefractive optics in dynamic interconnection,” Proc. IEEE 87, 2030-2049 (1999).

5. V. Coda, M. Chauvet, F. Pettazzi, and E. Fazio, "3-D integrated optical interconnect induced by self-focused beam," Electron. Lett. 42, 463-465 (2006).

6. S. Kawata, "Photorefractive optics in three-dimensional digital memory," Proc. IEEE 87, 2009-2020 (1999).

7. D. Staebler, W. Burke, W. Phillips, and J. Amodei, "Multiple storage and erasure of fixed holograms in Fe-doped $\mathrm{LiNbO}_{3}$," Appl. Phys. Lett. 26, 182-184 (1975).

8. Q. W. Song, C.-P. Zhang, and P. J. Talbot, "Self-defocusing, selffocusing, and speckle in $\mathrm{LiNbO}_{3}$ and $\mathrm{LiNbO}_{3}: \mathrm{Fe}$ crystals," Appl. Opt. 32, 7266-7271 (1993).

9. G. C.Valley, M. Segev, B. Crosignani, A. Yariv, M. M. Fejer, and M. C. Bashaw, "Dark and bright photovoltaic solitons," Phys. Rev. A 50, R4457-R4460 (1994).

10. E. Fazio, F. Renzi, R. Rinaldi, M. Bertolotti, M. Chauvet, W. Ramadan, A. Petris, and V. I. Vlad, "Screening-photovoltaic bright solitons in lithium niobate and associated single-mode waveguides,” Appl. Phys. Lett. 85, 2193-2195 (2004).
11. S. Juodkazis, V. Mizeikis, M. Sudzius, H. Misawa, K. Kitamura, S. Takekawa, E. G. Gamaly, W. Z. Krolikowski, and A. V. Rode, "Laser induced memory bits in photorefractive $\mathrm{LiNbO}_{3}$ and $\mathrm{LiTaO}_{3}$," Appl. Phys. A 93, 129-133 (2008).

12. Y. Gao, S. Liu, Z. Liu, and T. Song, "Transmission of digital images consisting of white-light dark solitons," Appl. Opt. 44, 6948-6951 (2005).

13. H. D. Wen, S. M. Liu, X. Z. Zhang, R. Guo, G. Q. Zhang, Q. Sun, J. J. Xu, and G. Y. Zhang, "Photorefractive phase mask," Proc. SPIE 4277, 303-310 (2001).

14. P. Günter and J. Huignard, eds., Photorefractive Effects and Applications (Springer-Verlag, 1988).

15. D. Psaltis, F. Mok, and H.-Y. Li, "Nonvolatile storage in photorefractive crystals," Opt. Lett. 19, 210-212 (1994).

16. P. Yeh, ed., Introduction to Photorefractive Nonlinear Optics (Wiley, 1993).

17. F. Devaux, V. Coda, M. Chauvet, and R. Passier, "New time-dependent photorefractive three-dimensional model: application to self-trapped beam with large bending," J. Opt. Soc. Am. B 25, 1081-1086 (2008).

18. F. Devaux and M. Chauvet, "Three-dimensional numerical model of the dynamics of photorefractive beam self-focusing in InP:Fe," Phys. Rev. A 79, 033823 (2009).

19. C. Johnson, ed., Matrix Theory and Applications (American Mathematical Society, 1990).

20. Y. Gao, S. Liu, X. Zhang, and Y. Lu, "White-light photorefractive phase mask," Appl. Opt. 44, 1533-1537 (2005). 